\title{
Evaluation of the diffusion coefficient of fluorine during the electropolishing of niobium
}

\author{
Hui Tian* and Charles E. Reece \\ Thomas Jefferson National Accelerator Facility, Newport News, Virginia 23606, USA
}

(Received 30 November 2009; published 25 August 2010)

\begin{abstract}
Future accelerators require unprecedented cavity performance, which is strongly influenced by interior surface nanosmoothness. Electropolishing (EP) is the technique of choice being developed for high-field superconducting radio frequency (SRF) cavities. Previous study has shown that the mechanism of $\mathrm{Nb}$ electropolishing proceeds by formation and dissolution of a compact salt film under fluorine diffusionlimited mass transport control. We pursue an improved understanding of the microscopic conditions required for optimum surface finishing. The viscosity of the standard electrolyte has been measured using a commercial viscometer, and the diffusion coefficient of fluorine was derived at a variety of temperatures from 0 to $50^{\circ} \mathrm{C}$ using a $\mathrm{Nb}$ rotating disk electrode. In addition, data indicate that electrode kinetics becomes competitive with the mass transfer current limitation and increases dramatically with temperature. These findings are expected to guide the optimization of EP process parameters for achieving controlled, reproducible, and uniform nanosmooth surface finishing of SRF cavities.
\end{abstract}

DOI: 10.1103/PhysRevSTAB.13.083502

PACS numbers: $82.45 . \mathrm{Rr}, 82.80 . \mathrm{Fk}, 81.65 . \mathrm{Ps}$

\section{INTRODUCTION}

Superconducting radio frequency (SRF) cavities have become the principal building blocks for charged particle accelerators. The unique characteristics of SRF materials allow them to support very high electromagnetic fields on their surfaces with minimal parasitic heat dissipation when cooled to low temperatures, typically $\sim 2 \mathrm{~K}$. New applications of SRF cavities are forthcoming for some of science's grandest instruments, such as Jefferson Lab's Continuous Electron Beam Accelerator Facility and its $12 \mathrm{GeV}$ upgrade now in progress, an envisioned electron ion collider, the Facility for Rare Isotope Beams, the International Linear Collider (ILC), proton-proton colliders, x-ray free electron lasers, storage ring light sources, linac-based light sources, energy recovery linacs, muon storage rings for neutrino sources, and eventually perhaps a high energy muon collider. These research tools are opening or will open new scientific frontiers, offering opportunities for revolutionary scientific breakthroughs.

Essential to the proper function of SRF accelerator cavities is obtaining pure, clean, and smooth interior surfaces, given that only the topmost $40-50 \mathrm{~nm}$ layer is directly involved with the rf fields. Controlling the creation and preservation of this finished surface is paramount. Electropolishing (EP) is the technique of choice being developed for high-field SRF cavities. With routine operation of EP facilities in recent years, more multicell SRF cavity test data have become available. Cavity performance variability has emerged as a major challenge for electropolishing processed niobium cavities [1,2], especially for the ILC, the application potential most visible

\footnotetext{
*Corresponding author. huit02@jlab.org
}

to the public, with 16000 nine-cell cavities intended to perform at historically high acceleration gradient $(>31 \mathrm{MV} / \mathrm{m})$. One outstanding variability issue is the unpredictable onset of field emission. Another is the quench field, which has been observed to vary unpredictably in electropolished cavities in the absence of field emission. Ongoing progress against field emission relies on elimination of surface adhering particles by improved cleanliness and high pressure rinsing. Quenches without $\mathrm{x}$-ray emission are usually associated with defects: foreign material inclusions, weld beads, etch pits, and mechanical damages are severe examples. At present, besides reducing the incidence of severe defect-induced quenches via improved quality control of manufacturing practice, a complete understanding of process parameters and adequate process control are very important to help achieve reproducible high gradient cavities.

Electropolishing is a surface finishing process based on anodic dissolution of a metal or alloy in an appropriately chosen electrolyte [3]. It is characterized by the elimination of microroughness (leveling) and the absence of crystallographic and grain boundary attack (brightening) and results in the production of smooth, bright surfaces $[4,5]$. Leveling results from the fact that protruding parts of a rough surface dissolve faster than recessed parts. This can be achieved under either Ohmic or mass transport control [5]. Brightening can only occur under mass transport control, which suppresses crystallographic etching [5-7]. The supply and removal of reactant and product contribute to three different forms of mass transport: (i) diffusion, defined as the movement of species due to concentration gradient; (ii) convection, in which the movement is due to external mechanical energy-for example, electrode rotation or density variation; and (iii) migration movement due to a potential gradient-that is, only charged species 
are affected [8]. The EP process typically now applied to cavities was inherited from Siemens Co. in the 1970s [9] and was further developed by KEK in collaboration with Nomura Plating [10]. Typically, a mixture of hydrofluoric (46\%-49\%) and sulfuric acids (95\%-98\%) by volume ratio of $1: 9$ or $1: 10$ is used with a temperature range of $30-45^{\circ} \mathrm{C}$ measured at the cavity surface, for example, the horizontal EP in Jefferson Lab and Deutsches Elektronen Synchrotron, or in the electrolyte, for example, the High Energy Accelerator Research Organization (commonly known as KEK) EP facility, a current density of $30-100 \mathrm{~mA} / \mathrm{cm}^{2}$, and an applied cell voltage of typically $10-20 \mathrm{~V}$. The surface area ratio of the niobium cavity (anode) to the typical high purity aluminum tube cathode is 10:1 during practical cavity EP processing of cylindrically symmetric multicell $\mathrm{Nb}$ SRF cavities.

Past cavity EP studies done on niobium SRF cavities have shown that the best polishing condition occurs in the current-limited plateau of the polarization curves. Parameters such as electrolyte temperature, acid concentration, viscosity, and stirring have strong effects on the EP process [11]. Some studies conducted on either small samples or half-cells tried to evaluate the influence of each parameter [12]. However, the application of electrochemical techniques is needed for the development of a clear picture of the exact role of each parameter involved during the EP process.

In our previous study, electrochemical impedance spectroscopy and related techniques point to the electropolishing mechanism of $\mathrm{Nb}$ in a sulphuric and hydrofluoric acid electrolyte as the growth and dissolution of a compact surface salt film, presumed to be $\mathrm{Nb}_{2} \mathrm{O}_{5}$, under the diffusion-limited mass transport control of fluoride and its related ions [13]. However, a detailed understanding of the diffusion of fluorine and a parametrization of resulting surface finishing control is still missing.

Rotating disk electrode (RDE) techniques are frequently used in electrochemical kinetic experiments to establish specific momentum and mass transfer characteristics $[8,14,15]$. RDE takes the form of a $\sim 5 \mathrm{~mm}$ diameter polished disk surrounded by an insulation sheath on all the sides except one exposed face. The structure is rotated rapidly about an axis perpendicular to the surface of the disk electrode; this movement of rotation creates a welldefined solution flow pattern as illustrated in Fig. 1 [8,14], in which the mass transport of limiting species is deliberately dominated by convection $[8,14]$. This property allows the RDE to be used to calculate parameters related to mass transport, such as the diffusion coefficient of various electroactive species. The hydrodynamics of the RDE system have been solved analytically by assuming spatially constant transport properties [16]. By solving the convective diffusion equation with the boundary conditions of the hydrodynamic velocity profile, the Levich equation [Eq. (1)] can be used to describe the relationship of limiting

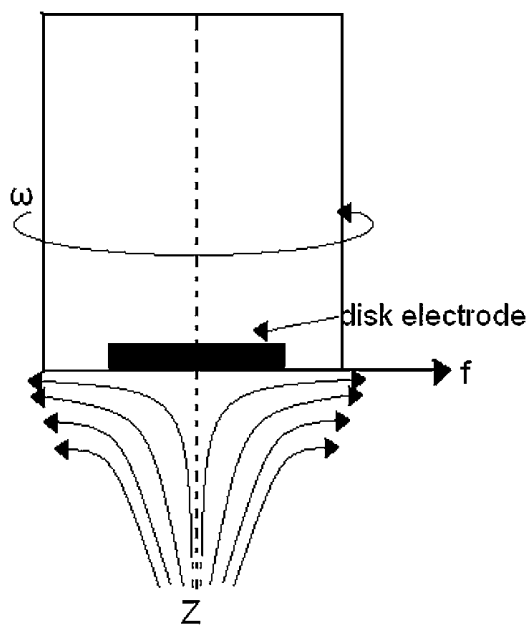

FIG. 1. Solution movement caused by rotation of an RDE [8].

current density $j_{L}$, to the physical properties of the electrolyte bath [7,17-20]:

$$
j_{L}=0.62 n F D^{0.67} \nu^{-0.166} c \omega^{0.5} .
$$

Here, $j_{L}$ is the limiting current density contributed by convective diffusion only, $n$ is the number of electrons involved in electrode reaction (for fluoride and its related ions $n$ equals 1 ), $F$ is the Faraday constant, $96485 \mathrm{Cmol}^{-1}, \nu$ is the kinematic viscosity in $\mathrm{m}^{2} \mathrm{~s}^{-1}, c$ is the concentration of electroactive species in bulk solution in mol. $\mathrm{m}^{-3}$ (the concentration of fluoride in 1:10 $\mathrm{HF} / \mathrm{H}_{2} \mathrm{SO}_{4}$ studied here is $2670 \mathrm{~mol} \mathrm{~m}^{-3}$ ), and $\omega$ is the rotation speed in $\operatorname{rad~s}^{-1}$. Note that

$$
\text { slope }\left(j_{L} \text { vs } \omega^{0.5}\right)=0.62 n F D^{0.67} \nu^{-0.166} c \text {. }
$$

Hence, a test for pure mass transport control is that the plot of $j_{L}$ vs $\omega^{0.5}$ is linear and passes through the origin [21]. The corresponding diffusion coefficient $D\left(\mathrm{~m}^{2} \mathrm{~s}^{-1}\right)$ can then be determined when the kinematic viscosity of electrolyte and mass limited species concentration are known. The kinematic viscosity of a fluid is simply the dynamic viscosity divided by its density. We measured the density of the electrolyte in this study to be $1760 \mathrm{~kg} / \mathrm{m}^{3}$. Table I

TABLE I. The measured dynamic viscosity of 1:10 $\mathrm{HF} / \mathrm{H}_{2} \mathrm{SO}_{4}$ electrolyte and calculated diffusion coefficient of fluorine at $1^{\circ} \mathrm{C}, 9^{\circ} \mathrm{C}, 19^{\circ} \mathrm{C}, 30^{\circ} \mathrm{C}, 41^{\circ} \mathrm{C}$, and $50^{\circ} \mathrm{C}$.

\begin{tabular}{ccc}
\hline \hline $\begin{array}{c}\text { Temperature } \\
\left({ }^{\circ} \mathrm{C}\right)\end{array}$ & $\begin{array}{c}\text { Dynamic viscosity } \\
(\mathrm{cP})\end{array}$ & $\begin{array}{c}\text { Diffusion coefficient } \\
\left(\mathrm{m}^{2} / \mathrm{s}\right)\end{array}$ \\
\hline 1 & 45 & $2.69 \times 10^{-12}$ \\
9 & 38 & $4.86 \times 10^{-12}$ \\
19 & 29 & $8.77 \times 10^{-12}$ \\
30 & 22 & $1.29 \times 10^{-11}$ \\
41 & 20 & $2.83 \times 10^{-11}$ \\
50 & 18 & $2.90 \times 10^{-11}$ \\
\hline \hline
\end{tabular}

${ }^{\mathrm{a}}$ The dynamic viscosity was calibrated against reference standard with and without guardleg in place. 


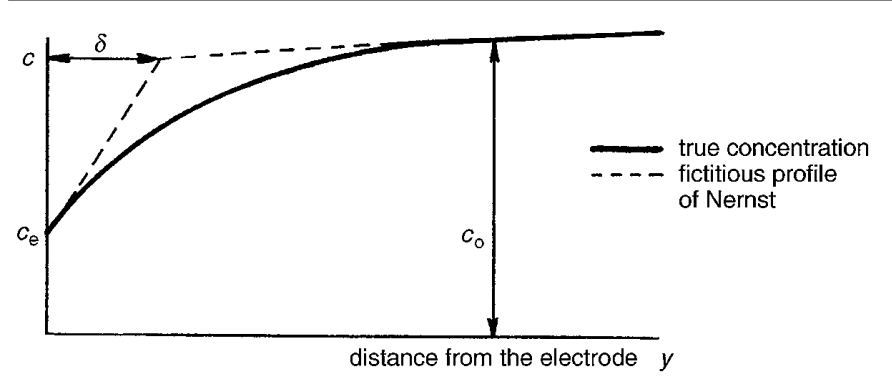

FIG. 2. Representation of the Nernst diffusion layer profile model [8].

lists the measured dynamic viscosity, and calculated diffusion coefficient of fluorine in the EP solution under different temperatures.

According to the Nernst diffusion layer model, the electrolyte can be divided into two zones (see Fig. 2) [8]. The first region is closest to the surface of the electrode with the thickness $\delta$, which is named the Nernst diffusion layer, where it is assumed that the fluid is totally stagnant and thereby diffusion is the only mode of mass transport. In the second region outside the Nernst diffusion layer, a strong convection occurs and all species concentrations are considered as constant. The model thus assumes a concentration profile perpendicular to an electrode surface corresponding to the dotted straight lines of Fig. 2. The thickness $\delta$ of this layer is called the effective (or equivalent) thickness of the diffusion layer. Its definition is apparent from Fig. 2. It is the thickness which the diffusion layer would have if the concentration profile were a straight line coinciding with the tangent to the true concentration profile at the interface, and that straight line were extended up to the point where the bulk concentration is reached. The diffusion-limited current density is then given by [8]

$$
J_{l}=n F D c / \delta
$$

So, for a given diffusion-limited current density, fluorine concentration, and measured diffusion constant, one can calculate the effective thickness of the diffusion layer.

Recent studies for different composition of hydrofluoric and sulfuric acid electrolytes done by Fabien Éozénou et al. suggested that the diffusion coefficient of fluorine is lower in the aged mixtures by assuming the concentration of fluorine was constant [22]. In this study, we report determination of the diffusion coefficient of fluoride and its related ions in 1:10 mixture of EP solution using a $\mathrm{Nb}$ rotating disk electrode at different temperatures. The thickness of the Nernst diffusion layer at different temperatures was determined, and those values were used to explore the optimization of EP process parameters for achieving uniform nanosmooth surface finishing.

\section{EXPERIMENTAL STUDIES}

RDE experiments were performed on high purity polycrystalline $\mathrm{Nb}$ disk electrode samples with a reactive surface area of $0.19 \mathrm{~cm}^{2}$. The $\mathrm{Nb}$ disks were mechanically polished up to 4000 grit emery paper, ultrasonically rinsed with distilled water and isopropanol, and air dried. A high purity $\mathrm{Al}$ wire was used as the counterelectrode. A Gamry RDE710 rotating disk electrode setup was used for the experiments with each sample mounted in a Teflon holder. The reference electrode was a saturated mercury/mercurous sulphate electrode (MSE). The electrolytes were prepared from analytic grade 49\% hydrofluoric (J. T. Baker) and $96 \%$ sulphuric acid (J. T. Baker) with the volume ratio of $1: 10$.

The polarization curves were measured stepwise (step size $=0.1$ volts, dwell time $=0.25$ second) over the potential range from 0 to 22 volts. The potential between the $\mathrm{Nb}$ electrode and the reference electrode was recorded using a Keithley 6517A electrometer. The addition of the reference electrode allows monitoring of the potentials on the cathode and anode independently. The current was recorded with a Keithley 2000 multimeter. Prior to the polarization measurements, the electrodes were immersed in the electrolyte in an open circuit condition for 3 to 5 minutes. In order to maintain a stable temperature during the measurement, the electrochemical cell was immersed in a water bath with constant circulation of controlled proportions of hot $\left(\sim 70^{\circ} \mathrm{C}\right)$, cold $\left(\sim 15^{\circ} \mathrm{C}\right)$, and ice water $\left(\sim 0^{\circ} \mathrm{C}\right)$. Two thermocouples were used to monitor the temperature of the electrolyte. One was placed midway between the anode and cathode to record the temperature of the bulk electrolyte, the other was positioned inside of the water bath.

The electrolyte viscosities were measured at different temperatures with a Brookfield DV - II + PRO digital viscometer without stainless steel guardleg. The dynamic viscosity was calibrated against a viscosity standard. The variation of repeated viscosity measurements was about $1.5 \%$. The electrolyte density was measured by weighing the electrolyte in a Teflon volumetric flask. The reported diffusion coefficients were verified by repeated experiments, with typical variations in limiting current densities of about $2 \%$.

\section{EXPERIMENTAL RESULTS AND DISCUSSIONS}

Figure 3 shows anode polarization curves of an $\mathrm{Nb}$ disk electrode for 1:10 volume ratio of $\mathrm{HF}$ and $\mathrm{H}_{2} \mathrm{SO}_{4}$ electrolyte at different angular velocities at $9^{\circ} \mathrm{C}$. The same anode polarization curves have been also obtained at the other temperatures studied here, such as $1^{\circ} \mathrm{C}, 19^{\circ} \mathrm{C}, 30^{\circ} \mathrm{C}, 41^{\circ} \mathrm{C}$, and $50^{\circ} \mathrm{C}$. The electrolyte temperature during the measurement was regulated within $50^{\circ} \mathrm{C}$. All the polarization curves exhibit a well-defined and wide potential range of 


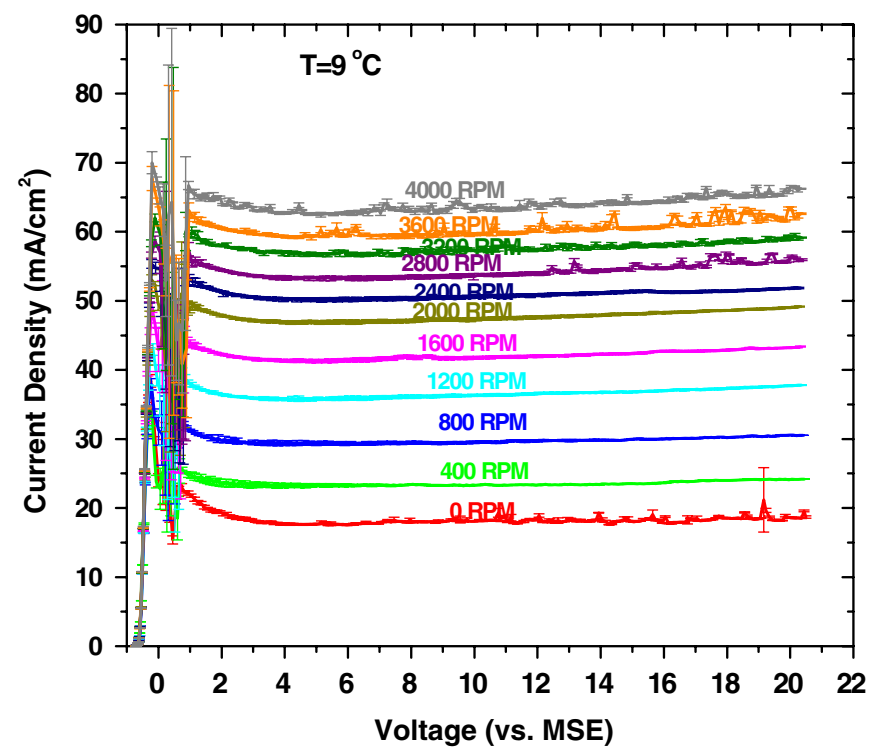

FIG. 3. (Color) The anode polarization curves of $\mathrm{Nb} \mathrm{RDE}$ in 1:10 volume ratio $\mathrm{HF}$ and $\mathrm{H}_{2} \mathrm{SO}_{4}$ at $9^{\circ} \mathrm{C}$.

limiting current density plateau. In this range the current is independent of the polarization voltage.

Figure 4 shows how the limited current density varies with the different RDE angular velocities at $19^{\circ} \mathrm{C}$. Similar measurements were made under the other controlled temperature conditions. Figure 5 shows the typical dependence of the limiting current density on the square root of RDE rotation rate [23]. The variation of the limiting current density shows a linear behavior with respect to $\omega^{1 / 2}$, which provides distinct evidence that the supply of reactant, such as $F$ ion or its related species ( $\mathrm{HF}^{-}$or HF [15]), is under mass transport control in a media with uniform viscosity.

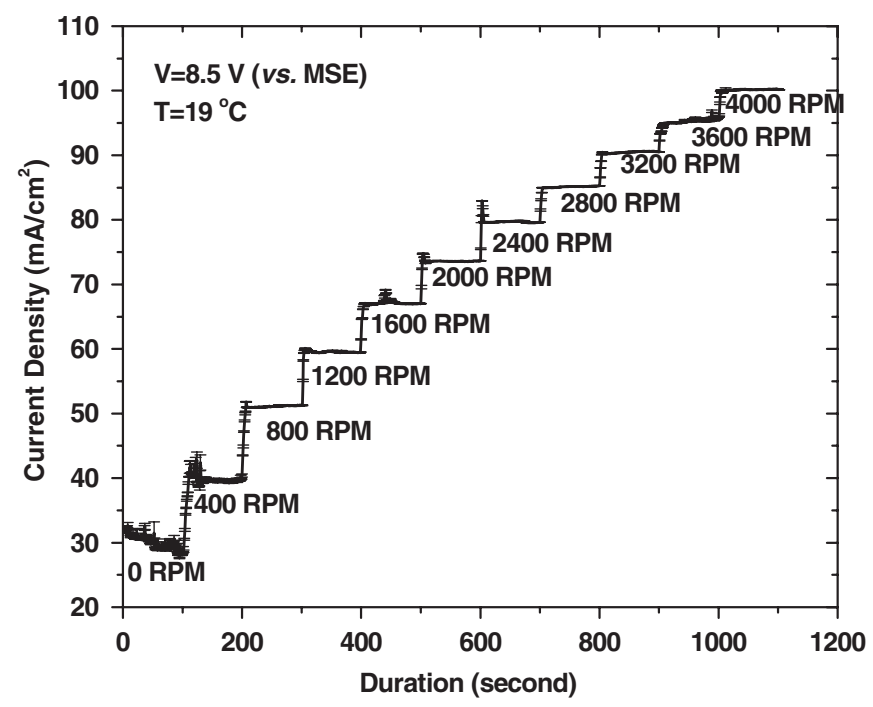

FIG. 4. The variation of limiting current density of $\mathrm{Nb}$ disk electrode with different rotation rate in 1:10 volume ratio $\mathrm{HF}$ and $\mathrm{H}_{2} \mathrm{SO}_{4}$ at $19^{\circ} \mathrm{C}$ at a potential $8.5 \mathrm{~V}$ (vs MSE).

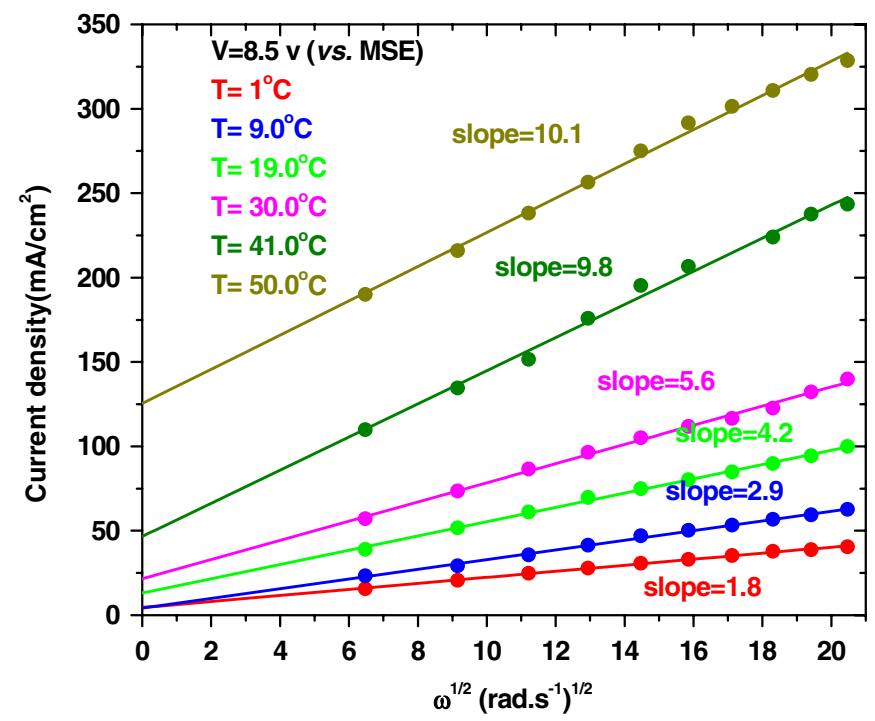

FIG. 5. (Color) The limiting current density of $\mathrm{Nb}$ disk electrode in 1:10 volume ratio $\mathrm{HF}$ and $\mathrm{H}_{2} \mathrm{SO}_{4}$ plotted as a function of the square root of rotation rate at a potential $8.5 \mathrm{~V}$ (vs MSE).

However, the nonzero offsets at the higher electrolyte temperatures indicate the presence of an electrode kinetic mechanism; we assume it to be an electrochemical etching process that proceeds in parallel with the diffusion-limited mass transport and note that its contribution to the plateau current increases dramatically with electrolyte temperature.

In this study, $J_{L}$ is determined by subtracting the nonzero offsets in Fig. 5 from the measured limiting current density under static condition at $8.5 \mathrm{~V}$ anode potential for different temperature electrolytes studied here. The calculated Nernst diffusion layer thickness according to Eq. (3)

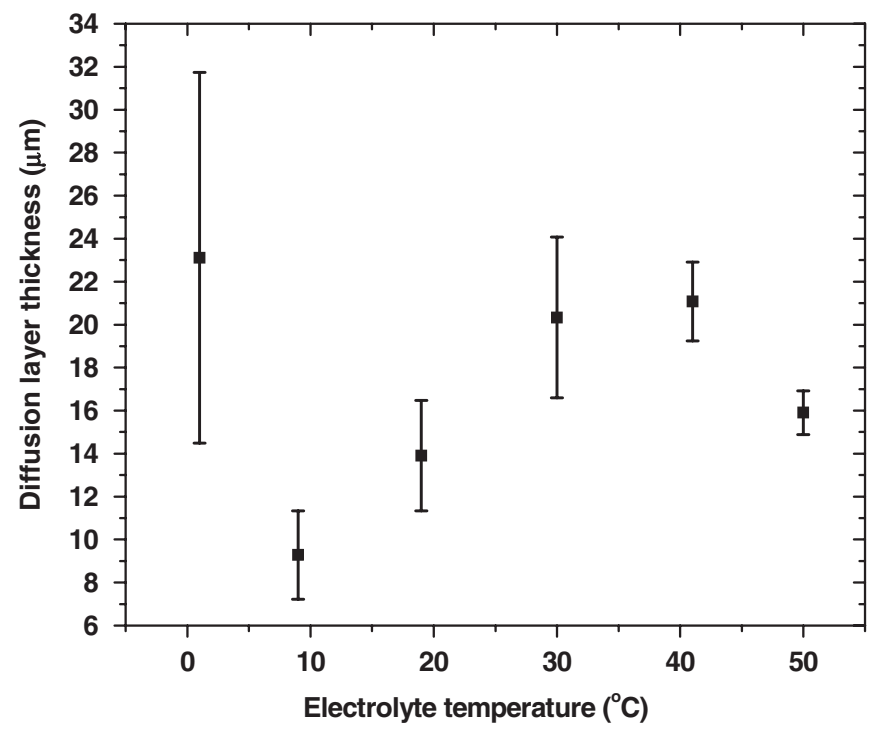

FIG. 6. The effective Nernst diffusion layer thickness of fluorine in 1:10 volume ratio $\mathrm{HF}$ and $\mathrm{H}_{2} \mathrm{SO}_{4}$ during diffusion $\mathrm{EP}$ of niobium at the different temperatures. 
is shown in Fig. 6. The uncertainty of the Nernst diffusion layer thickness determination under each temperature mostly comes from the variations of the diffusion-limited current density and the uncertainty of subtraction of the offset (especially for the low temperature, such as $1^{\circ} \mathrm{C}$ ). Note that the Nernst diffusion layer thickness is maintained about $10-20 \mu \mathrm{m}$ over the temperature range $1-50^{\circ} \mathrm{C}$ for the electrolyte studied here. At this time, we offer no interpretation for the temperature dependence of Nernst diffusion layer suggested by these data. However, it is important for our application to understand that it is the concentration gradient on the scale of $\delta$ that is expected to produce the most optimum leveling effect.

Figures 7(a) and 7(b) show atomic force microscopy (AFM) topography of two $\mathrm{Nb}$ samples centrifugal barrel polished (CBP) by KEK. We label them as rough CBP and
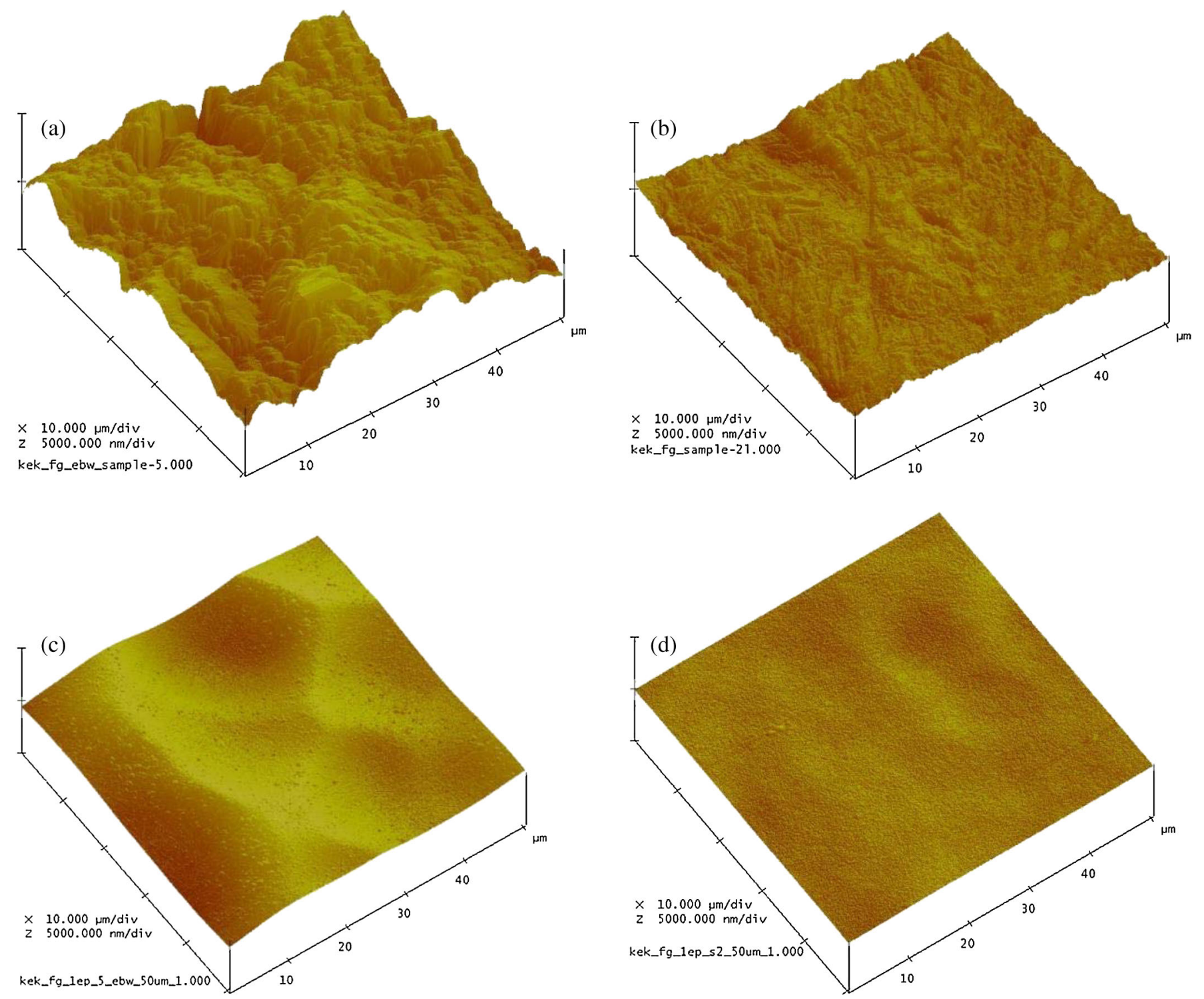

FIG. 7. (Color) AFM images of CBP polycrystalline $\mathrm{Nb}$ samples before and after 100 min of electropolishing at $30 \pm 1^{\circ} \mathrm{C}$ with $14 \mathrm{~V}$ applied cell voltage: (a) rough $\mathrm{CBP}$, (b) fine CBP, (c) rough CBP after electropolishing, and (d) fine CBP after electropolishing. 
$1.6 \mu \mathrm{m}$ for the fine CBP sample. After $100 \mathrm{~min}$ of EP polishing $(30 \mu \mathrm{m}$ removal) by $1: 10$ volume ratio $\mathrm{HF} / \mathrm{H}_{2} \mathrm{SO}_{4}$ at $30 \pm 1{ }^{\circ} \mathrm{C}$ with 14 volts applied cell voltage with cathode/anode area ratio of 2:1, the fine CBP surface achieves a better finish, with $55 \pm 5 \mathrm{~nm}$ mean square root roughness [see Fig. 7(d)], and the surface variation is about $150 \mathrm{~nm}$, as compared with the rough CBP, where $R_{q}$ is $187 \pm 22 \mathrm{~nm}$ and $R_{z}$ is about $1.6 \mu \mathrm{m}$ [Fig. 7(c)]. These results give strong evidence that diffusion-limited mass transfer would produce the nanosmooth surface finishing. Further refinement of the polishing analysis as a function of temperature is planned in the near future.

Figure 8 shows the power spectral density (PSD) analysis results of fine CBP treated polycrystalline, large grain, and single crystal $\mathrm{Nb}$ samples with $100 \mathrm{~min} \mathrm{EP}$ at $30-1{ }^{\circ} \mathrm{C}$ with 14 volts applied cell voltage with cathode/anode area ratio of 2:1. The PSD-based description of surfaces was recently developed for $\mathrm{Nb}$ surface topography study under cavity production conditions [25-27]. It is a method of combining the measurements from different diagnostic instruments, and also reducing instrumental effects when one measures parameters such as surface roughness and correlation length $[28,29]$. PSD represents the spatialfrequency spectrum of surface roughness measured in inverse-length units. Briefly, a series of AFM and stylus profilometry scans are obtained from several surface locations of the specimens according to the protocol developed, and the squares of the Fourier transforms of the measured surface heights are combined as a plot of roughness PSD versus spatial frequency (inverse of wavelength). The PSD analysis in Fig. 9 shows that fine CBP produces a very reproducible surface for polycrystalline, large grain, and single crystal $\mathrm{Nb}$. However, after $100 \mathrm{~min} \mathrm{EP}$ at $30-1{ }^{\circ} \mathrm{C}$, a

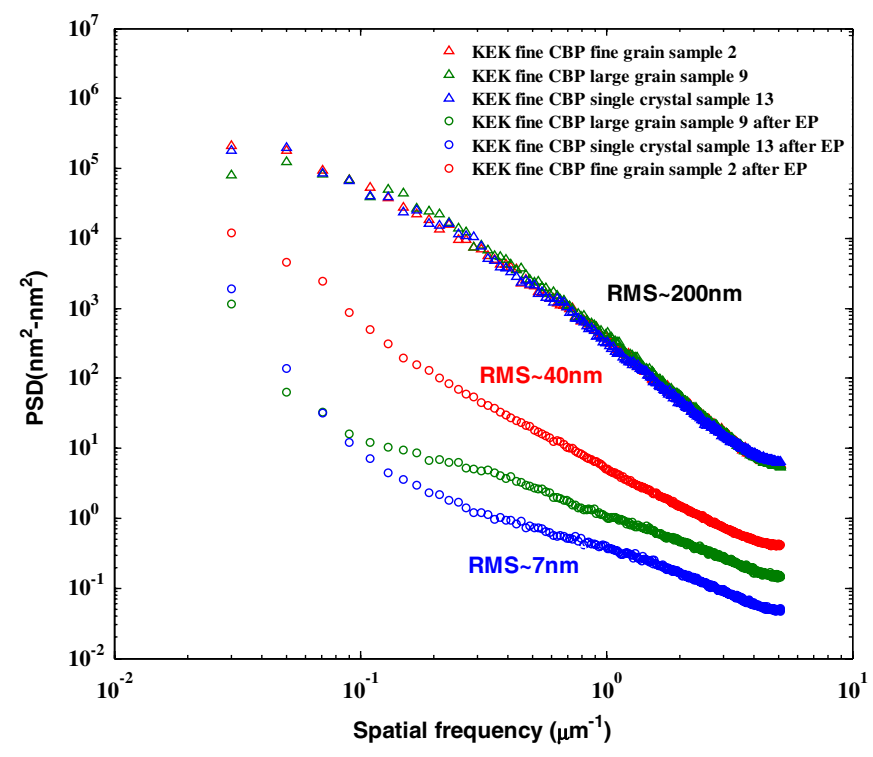

FIG. 8. (Color) The PSD of fine CBP polycrystalline, large gain and single crystal $\mathrm{Nb}$ samples after $30 \mu \mathrm{m}$ removal by EP at $30 \pm 1^{\circ} \mathrm{C}$ with $14 \mathrm{~V}$ applied cell voltage. noticeable difference was observed from the different crystalline $\mathrm{Nb}$ samples. Compared with fine grain $\mathrm{Nb}$ samples, the single crystal sample shows the smallest amplitude of PSD and better correlation length. We explain the difference as perhaps caused by electrochemical etching occurring in parallel with the desired EP brightening process at the regulated $30^{\circ} \mathrm{C}$ condition, as suggested by the nonzero offset in the current density vs square root of rotation rate curves. If the interpretation is correct, one would anticipate a reduction in material dependent topography as the EP process temperature is lowered. Further studies will test this hypothesis.

\section{CONCLUSION}

In this study, $\mathrm{Nb}$ electropolishing was studied using a rotating disk electrode in a 1:10 volume ratio of $\mathrm{HF} / \mathrm{H}_{2} \mathrm{SO}_{4}$ electrolyte at different temperatures. The limiting current density demonstrates a clear dependence on fluorine diffusive mass transport. However, a parallel electrode kinetics process has been identified and it strongly depends on temperature. This suggests that high temperature may activate an etching process that recognizes different grain orientations, resulting in a nonuniform surface finishing instead of brightening. The viscosity of the standard $\mathrm{HF} / \mathrm{H}_{2} \mathrm{SO}_{4}$ electrolyte was measured and found to strongly depend on the electrolyte temperature. The analysis yields an effective diffusion coefficient for fluoride ion on the order of $10^{-12}-10^{-11} \mathrm{~m}^{2} \mathrm{~s}^{-1}$ under the temperatures studied, yielding a Nernst diffusion layer thickness of $10-20 \mu \mathrm{m}$. This study is expected to improve the fundamental understanding needed to guide the optimization of EP of niobium for SRF cavities required for particle accelerators.

\section{ACKNOWLEDGMENTS}

This research was conducted at Thomas Jefferson National Accelerator Facility in collaboration with the College of William and Mary, Department of Applied Science, for the U.S. Department of Energy under Contract No. DE-AC05-06OR23177. This manuscript has been authored by Jefferson Science Associates, LLC under U.S. DOE Contract No. DE-AC05-06OR23177.

[1] C. Ginsburg, http://ilcagenda.linearcollider.org/ conferenceDisplay.py? confId $=1855$.

[2] R. L. Geng, Jefferson Lab Internal Report.

[3] W. G. Cochran, Proc. Cambridge Philos. Soc. 30, 365 (1934).

[4] R. Sautebin, H. Froidevaux, and D. Landolt, J. Electrochem. Soc. 127, 1096 (1980).

[5] D. Landolt, Electrochim. Acta 32, 1 (1987).

[6] H. Abrams and C.L. Mantell, Electrochem. Technol. 5, 287 (1967). 
[7] M. Matlosz, S. Magaino, and D. Landolt, J. Electrochem. Soc. 141, 410 (1994).

[8] A. J. Bard and L. R. Faulkner, Electrochemical Methods (Wiley, New York, 1980).

[9] H. Diepers, O. Schmidt, H. Martens, and F. S. Sun, Phys. Lett. A 37, 139 (1971).

[10] K. Saito, Y. Kojima, T. Furuya, S. Mitsonobu, S. Noguchi, K. Hosoyama, T. Nakazato, T. Tajima, K. Asano, K. Inoue, Y. Iino, H. Nomura, and K. Takeuchi, in Proceedings of the 4th Workshop on RF Superconductivity (KEK, Tsukuba, Japan, 1989), Vol. 2, p. 635.

[11] V. Palmieri, in Proceedings of the 11th SRF Workshop (DESY, Travemünde/Lübeck, Germany, 2003), WeT02 [http://srf2003.desy.de/fap/].

[12] F. Eozénou, A. Aspart, C. Antoine, and B. Maliki, CARE Report No. 06-10-SRF, 2006.

[13] H. Tian, S. G. Corcoran, C. E. Reece, and M. J. Kelley, J. Electrochem. Soc. 155, D563 (2008).

[14] R.N. Adams, Electrochemistry at Solid Electrodes (Dekker, New York, 1969).

[15] S. Cattaria, M. Masiani, and B. Tribollet, J. Electrochem. Soc. 149, B457 (2002).

[16] R. Sautebin and D. Landolt, J. Electrochem. Soc. 129, 946 (1982).

[17] V. G. Levich, Physicochemical Hydrodynamics (PrenticeHall, New York, 1962).

[18] R. Vidal and A. C. West, J. Electrochem. Soc. 142, 2689
(1995).

[19] B. Du and I. I. Suni, J. Electrochem. Soc. 151, C375 (2004).

[20] B. Du and I. I. Suni, J. Appl. Electrochem. 34, 1215 (2004).

[21] J.H. Han, A. Bowen, T.N. Andryushchenko, R.P. Chalupa, A. E. Miller, H. S. Simka, K. C. Cadien, and S. Shankar, J. Appl. Electrochem. 38, 1 (2007).

[22] F. Éozénou, S. Berry, Y. Gasser, and J-P. Charrier, in 14th International Conference on RF Superconductivity (HZB, Berlin, Germany, 2009), THPPO069 [http://accelconf. web.cern.ch/AccelConf/srf2009/index.htm].

[23] J. L. Hudson and T. T. Tsotsis, Chem. Eng. Sci. 49, 1493 (1994).

[24] T. Higuchi, K. Saito, Y. Yamazaki, T. Ikeda, and S. Ohgushi, in Proceedings of the 10th Workshop on RF Superconductivity (KEK, Tsukuba, Japan, 2001), p. 279.

[25] H. Tian, G. Ribeill, M.J. Kelley, and C. E. Reece, in Proceedings of the 13th SRF Workshop (Beijing University, Beijing, China, 2007), WEP04 [http://web5. pku.edu.cn/srf2007/proceeding.html].

[26] H. Tian, Ph.D. dissertation, Department of Applied Science, College of William and Mary, 2008.

[27] H. Tian, C. E. Reece, and M. J. Kelley (unpublished).

[28] J. Ferré-Borrull, A. Duparré, and E. Quesnel, Appl. Opt. 40, 2190 (2001).

[29] O. Vatel, Jpn. J. Appl. Phys. 32, 5671 (1993). 